Ocula ${ }^{23}$

Occhio semiotico sui media | Semiotic eye on media

www.ocula.it • ISSN 1724-7810 • Vol 21, No 23 (July 2020) • DOI: 10.12977/ocula2020-28

Fiori dell'anima. La simbologia dei fiori nell'immaginario religioso Flowers of the soul. The symbolism offlowers in the religious imagination a cura di Marco Papasidero e Francesco Galofaro

\title{
La rosa di Sharon \\ Fiori e piante nella tradizione ebraica antica
}

Ugo Volli

Università degli Studi di Torino, IT

ugo.volli@unito.it

\begin{abstract}
In the texts of the Jewish tradition, starting from the Torah, there is an abundant lexicon of plant species, which testifies to its settlement in the countryside. But the lexicon of flowers is much more limited, probably due to the geographical and ecological location of Jewish society in a land as arid as Israel was and also for cultural and religious reasons. Most of these quotes belong to the Canticle of Canticles, where they have an erotic character. But an abundant metaphorical and symbolic production developed on them which greatly expanded its meaning. The paper studies these developments in the Talmud, Zohar and Jewish tradition.
\end{abstract}

\section{Keywords}

Flowers; Song of Songs; Menorah; Lily; Rose

\section{Sommario/Content}

1. Problemi preliminari

2. I fiori nella cultura ebraica

3. Shoshanah

4. Havatzelet

Bibliografia 


\section{Ocula ${ }^{23}$}

Vol 21, No 23 (July 2020) • DOI: 10.12977/ocula2020-28

Ugo Volli | La rosa di Sharon. Fiori e piante nella tradizione ebraica antica

\section{Problemi preliminari}

Metafore e simboli floreali sono diffuse in molte culture civili e religiose, fra cui anche quella ebraica. Prima di esaminare questo dominio di senso, bisogna però affrontare due questioni preliminari. Il primo è la ragione di questa larga diffusione, che pur non essendo universale, è presente in moltissime culture, come ha mostrato Goody (1993). Dalle ricerche del grande antropologo britannico emerge che l'apprezzamento dei fiori è una costante molto generale fra gli esseri umani, ma essa è limitata da due fattori. Una è la scarsa presenza di fiori vistosi in certe regioni: così Goody per esempio spiega l'assenza di "cultura dei fiori" nell'Africa subsahariana, ma questo discorso si può estendere chiaramente a molte società che sono sorte in ambienti semidesertici o stepposi, o vi si identificano fortemente, come quella ebraica.

L'altro fattore più significativo sul piano teorico consiste nel fatto che abbastanza spesso la "cultura dei fiori" è bloccata dalla censura di società che in genere disapprovano anche altre forme di piacere visivo, come le rappresentazioni di oggetti naturali e in particolare del corpo umano, ${ }^{1}$ gli ornamenti e i profumi. Questo accadde per esempio nei periodi di iconoclastia nel Cristianesimo, fra l'ottavo e il decimo secolo a Bisanzio e dopo la Riforma protestante nell'Europa settentrionale o nelle correnti islamiche più radicali.

Vale la pena di porsi comunque la nostra seconda questione preliminare, cioè il problema generale della predilezione per i fiori, che è un tratto della specie umana condiviso con buona parte degli uccelli e alcuni insetti. ${ }^{2} \mathrm{Vi}$ sono numerose teorie evoluzioniste su questo atteggiamento: Steven Pinker (1997) propone che l'affinità per i fiori sia radicata nell'evoluzione biologica della specie umana. Nelle savane dell'Africa i nostri lontani antenati avrebbero identificato i fiori come "precursori della crescita, segnando il sito di futura frutta, noci o tuberi per creature abbastanza intelligenti da ricordarli". In altre parole, fare attenzione ai fiori sarebbe stata una risposta adattativa che ha permesso agli ominidi di localizzare il cibo e quindi sopravvivere all'età riproduttiva:

La mia ipotesi è che [il piacere per i fiori] derivi da una combinazione di due fenomeni psicologici. Considerare i fiori come oggetti di bellezza e benessere, che determinano una preferenza dell'habitat umano, e regalarli come beni preziosi, di cui il cibo di alta qualità è l'esempio prototipico. Penso che entrambi siano universali e probabilmente si sono verificati all'inizio dell'evoluzione del moderno Homo sapiens.

Stephen Kaplan (1992) concorda: "In sostanza, si può costruire un convincente argomento evolutivo" intorno alle ragioni per cui amiamo i fiori. "È adattivo trovare fiori attraenti e preziosi, dal momento che voler rimanere dove sono mette chi ne è attratto in una posizione migliore per raccogliere il raccolto quando i frutti seguono". Da questa "biofilia" (Edward O. Wilson

1 Su questo tema si vedano anche Volli (2019), Goody (1997).

2 Per una discussione breve ma interessante, si veda Palewitz (2001). 


\section{Ocula ${ }^{23}$}

Vol 21, No 23 (July 2020) • DOI: 10.12977/ocula2020-28

Ugo Volli | La rosa di Sharon. Fiori e piante nella tradizione ebraica antica

1984), radicata nella costituzione della specie, deriverebbe il notevole paradosso per cui i fiori (o meglio le piante da cui essi sono prodotti) sarebbero capaci in un certo senso di manipolarci per indurci a preferirli (Pollan 2001), come accade nei numerosi casi di "coabitazione" biologica, o addirittura di simbiosi anche con gli animali domestici; ma questo caso avrebbe particolare importanza determinando non solo le nostre scelte estetiche ma anche la forma del paesaggio per favorirne la proliferazione.

Il primo problema preliminare, già accennato già nelle osservazioni di Goody, fa dipendere la possibilità di una cultura dei fiori e di un loro uso simbolico e religioso da questioni ecologiche, cioè da che tipo di specie vegetali si ritrovino nell'ambiente naturale e sociale di quella società, il che a sua volta è determinato sia dalla sua localizzazione geografica e dal clima che vi regna, sia dai risultati di pratiche di selezione e coltivazione che vi sono svolte e su cui vale il discorso già accennato della simbiosi fra specie umana e certe specie vegetali, non solo quelle coltivate direttamente per il cibo, ma anche quelle selezionate per i fiori.

Ma al di là del dato botanico vero e proprio delle specie presenti in un dato ambiente e in un tempo determinato, che è possibile ricostruire almeno in parte anche per le culture più antiche per mezzo di metodi archeologici come lo studio dei pollini fossili, vi è un filtro culturale da tener presente. Certamente in una società cresciuta nel deserto o in clima tropicale sarà praticamente impossibile apprezzare fiori che hanno bisogno di un clima del tutto diverso come i tulipani o i ciclamini. Ma anche rispetto a specie vegetali fiorite presenti in quegli ambienti come i cactus o le agavi di certi deserti, bisogna vedere se questa parte colorata ed effimera delle piante è presa in considerazione sul piano del senso e come. Un metodo utile, e forse il solo possibile, è vedere come i fiori sono tradotti culturalmente: in immagini e decorazioni architettoniche, quando vi sono, e soprattutto nel linguaggio, nel lessico e nelle metafore, nella misura in cui questi dati sono conservati nei testi o possono essere ricostruire con metodi filologici.

\section{I fiori nella cultura ebraica}

Il lessico biblico sulle specie vegetali è piuttosto ricco, comprendendo fra le 80 e le 120 unità. ${ }^{3}$ Troviamo citati fra l'altro l'uva e il mandorlo, l'acacia e il grano, il melo, i fagioli, le cipolle, i capperi e i limoni, i carrubi e la menta, le lenticchie e i noci, la palme i melograni e le querce: la maggioranza di queste piante sono alberi, spesso fruttiferi, oppure sono vegetali agricoli, il che non fa meraviglia, dato che il complesso di testi che costituiscono la Bibbia ebraica (il Tanakh) fu scritto in società agricole o pastorali. Anche nell'“immaginario delle origini" (Volli 2011) di Genesi 1 e 2, in mezzo alle acque, al firmamento, ai luminari e agli animali, hanno un posto significativo i vegetali, ma fra essi sono citate solo "le erbe, le piante che producono seme, gli alberi da frutto"

3 Per i due elenchi, con i riferimenti testuali e biologici si può consultare: <https://ww2.odu.edu/ lmusselm/plant/bible/allbibleplantslist.php>. 


\section{Dcula ${ }^{23}$}

Vol 21, No 23 (July 2020) • DOI: 10.12977/ocula2020-28

Ugo Volli | La rosa di Sharon. Fiori e piante nella tradizione ebraica antica

(Gen 1:11), "cespugli della steppa [...] erba" (Gen 2:5) "alberi attraenti per la vista e buoni da mangiare" (Gen 2:9). È interessante notare che qui, come in seguito sono gli alberi (a partire da quello fatale del bene e del male) e non i fiori a essere considerati portatori di bellezza.

La citazione della Genesi si riferisce al gan Eden (il giardino dell'Eden), spesso definito in seguito e non impropriamente "paradiso terrestre", perché "paradiso" (pardes) è un termine arrivato alla cultura europea da quella ebraica, ma che essa ha preso da un termine persiano, pairi-daeza, luogo recintato, composto di pairi, intorno, e daeza, muro, ${ }^{4}$ cioè in definitiva "giardino chiuso" in analogia a quanto è arrivato dall'Oriente in certi luoghi d'Europa nella forma di chiostri e patii. Ora il fatto è che in questa nozione di giardino l'elemento principale non sono i fiori, come nei "giardini all'italiana" risalenti al Rinascimento, ma gli alberi, insieme alle acque, ai cespugli, alle erbe, ai marmi. I fiori sono certamente graditi, ma non elemento centrale.

A proposito di pardes, vale la pena di notare qui che il termine è stato usato largamente nella tradizione ebraica per indicare i quattro livelli dell'interpretazione, usando come dispositivo mnemonico le iniziali di nomi di questi livelli (PaRDeS: Pshat o interpretazione letterale, Remez o metaforica, Drash o allegorica, Sod o esoterica: Volli 2019). Ma il nome non è rimasto senza conseguenze. Quel che ci può interessare qui infatti è che questo rapporto metaforico fra giardino e interpretazione viene usata e sviluppata in un celebre brano tradizionale sui pericoli dell'esoterismo, in Talmud Babli, trattato "Chaghiga" 14b:

Cosi hanno insegnato i nostri saggi: quattro persone sono entrate nel Pardes ed erano: Ben Azai, Ben Zoma, Acher e Rabbi Akiva. Rabbi Akiva disse loro: quando arriverete alle pietre di marmo bianco non dite: acqua! Acqua!, dato che è scritto: colui che dice menzogne non potrà stare davanti ai miei occhi. Ben Azai guardò e morì, e di lui il verso dice: preziosa agli occhi di Dio è la morte dei suoi pii. Ben Zoma guardò e rimase ferito, e di lui dice il verso: hai trovato miele, smetti di mangiarne, o altrimenti ti sazierà al punto di vomitarlo. Acher si mise a tagliare i virgulti. Rabbi Akiva uscì in pace.

Come si vede anche nel giardino dell'interpretazione vi sono acque e marmi e miele e "virgulti", cioè erbe appena spuntate, che stanno metaforicamente per i giovani, ma non si parla di fiori. Insomma nel lessico botanico biblico e talmudico i fiori non sono né numerosi né preminenti.

Del resto un'assenza analoga va notata anche nella letteratura greca delle origini, dove l'Odissea dà rilievo quasi solo ai fiori commestibili del loto (IX, 92-102) ai prati inferi di asfodeli (XI, 539, 573) e al mitico "Moli" (X, 302306), fiore ricco di poteri magici che salva Ulisse da Circe, ma per il resto si limita a citazioni generiche, mentre Esiodo ne nomina diversi, sempre però nell'ambito di un paesaggio agricolo. Non che la bellezza dei fiori sia assente,

4 Cfr. Bernheim \& Stavvrides (1991); per l'etimologia <https://www.etimo. it/?term=paradiso $>$. 


\section{Dcula ${ }^{23}$}

Vol 21, No 23 (July 2020) • DOI: 10.12977/ocula2020-28

Ugo Volli | La rosa di Sharon. Fiori e piante nella tradizione ebraica antica

ma essa è una parte della natura, per di più molto fragile, che dà anzi luogo a una frequente metafora della morte, il "topos del fiore reciso". 5

Tornando alla tradizione ebraica, nel celebre passo (Deut 7: $8 \mathrm{sgg}$.) in cui Mosè descrive al popolo ebraico la "buona terra" che Dio ha promesso loro, oltre a parlare di sorgenti e minerali, di latte e di miele, la caratterizza come il luogo dove crescono alcuni vegetali importanti, che nella tradizione ebraica hanno preso poi per antonomasia il nome di "Sette specie": «una terra di grano e orzo, di viti, fichi e melograni, una terra di ulivi e miele» [che va probabilmente interpretato non come il prodotto delle api ma come il contenuto dei datteri, e dunque viene riferito alla palma].

Nessuno di questi vegetali produce fiori particolarmente notevoli, ma essi erano usati nelle offerte di primizie prescritte per la festa di Shavuot (Pentecoste), che è l'occasione canonica in cui tradizionalmente le sinagoghe sono adornate con fiori. La prima menzione di questa usanza è del rabbino Yaakov ben Moshe Halevi Moelin, noto come Maharil (XIV secolo), che scrive che "era usanza diffondere erba e fiori profumati sul pavimento della sinagoga in onore della sinagoga per festeggiare Shavuot" (Maharil 1565, ad vocem). Tuttavia, l'usanza può risalire addirittura all'esilio babilonese: il rabbino Chaim Yosef David Azulai, noto come Chida (Gerusalemme, 1724-1806), cita un antico Midrash che allude a questo l'usanza praticata al tempo dell'impero persiano.

Nelle interpretazioni tradizionali vengono date diverse ragioni per quest'uso, come il miracolo della sopravvivenza delle greggi durante i quaranta anni di viaggio dell'Esodo, che presuppone l'esistenza di pascoli, magari fioriti; o il giudizio divino che verrebbe fatto in questa data sulla fruttuosità degli alberi, o anche il verso del Cantico dei cantici in cui si parla di una "rosa tra le spine", che discuterò in seguito. ${ }^{6} \mathrm{Ma}$ è bene ricordare che Shavuot, come altre ricorrenze religiose, oltre al suo significato storico/teologico di ricordo della rivelazione del Sinai ha anche il carattere di un'antica festa agricola dedicata alle primizie (di qui il nome Yom HaBikkurim), il che riemerge in molti costumi caratteristici di questa ricorrenza, come quello di consumare in esso latticini.

Le sette specie ritornano poi fra i vegetali che si consumano nel seder (cena rituale) della festività di Tu Bishvat (il quindicesimo giorno del mese di Shevat, che cade fra gennaio e febbraio), detto Rosh Hashanà Lailanot (capodanno degli alberi), uno delle quattro date che il Talmud definisce come inizio di certe attività annuali (e dunque "capodanni") e discute nel trattato intitolato per l'appunto Rosh Hashanà ("Capo d'anno") alle pag. 14a-14b. Nel Talmud Tu Bishvat serve a determinare la scadenza annuale delle decime dei prodotti vegetali e costituisce la data a partire dalla quale termina la proibizione di raccogliere i frutti degli alberi nei loro primi tre anni di vita. Oggi essa celebra

5 Cfr. Celentano (1991), <http://illuminati.it/it/lett-latina-e-comparata/lezionie-conferenze/107-la-similitudine-del-fiore-reciso.html>, Gagliardi (2007).

6 Per l'espressione di queste ragioni tradizionali, rimando a <https://www. chabad.org/library/article_cdo/aid/2159/jewish/7-Classic-Reasons-for-ShavuotFlowers-and-Greenery.htm>. 


\section{Ocula ${ }^{23}$}

Vol 21, No 23 (July 2020) • DOI: 10.12977/ocula2020-28

Ugo Volli | La rosa di Sharon. Fiori e piante nella tradizione ebraica antica

il rapporto degli ebrei non solo col mondo vegetale, ma con la terra, in particolare quella di Israele. È d'uso piantare alberi e mangiare piatti soprattutto vegetali e bene auguranti, fra cui le Sette specie (Bachbout 1986).?

In realtà, l'uso dei fiori per scopi decorativi è un'idea relativamente moderna. Dai tempi biblici fino all'età moderna, il valore principale attribuito ai fiori era il loro profumo. Il profumo ha un significato molto importante nella cultura ebraica, ricostruito con molto acume da Corradini (2018). Anche nella società occidentale, l'uso di tenere fiori in casa e di regalarli è chiaramente connesso al loro profumo (Corbin 1982).

La raccolta di fiori è menzionata nella Bibbia una sola volta in un testo certamente tardo come il Cantico dei Cantici (6: 2): "La mia amata è andata nel suo giardino, ai letti di spezie, per nutrire i giardini e per raccogliere gigli". Qualche altro riferimento alla bellezza dei fiori non manca nella Bibbia ebraica. Innanzitutto va citato un brano profetico di Osea (14:4-7) in cui si parla di un futuro radioso per il popolo di Israele. Dice il profeta a nome di Dio:

4 Io guarirò la loro infedeltà, io li amerò di cuore, poiché la mia ira si è distolta da loro.

5 Io sarò per Israele come la rugiada;

egli fiorirà come il giglio

e spanderà le sue radici come il cedro del Libano.

6 I suoi rami si estenderanno;

la sua bellezza sarà come quella dell'ulivo

e la sua fragranza come quella del cedro del Libano.

7 Quelli che abiteranno alla sua ombra

faranno di nuovo crescere il grano

e fioriranno come la vite;

saranno famosi come il vino del Libano.

\section{Shoshanah}

Nella Bibbia sono menzionati per nome solo tre tipi di fiori. Il più diffuso nel testo ${ }^{8}$ si chiama shoshan o shoshanah: una parola che oggi designa la rosa, oltre a essere un diffuso nome comune il cui calco nelle lingue occidentali è "Susanna". Viene però generalmente tradotta come "giglio", per esempio nella citazione qui sopra, dato che non può essere una rosa, fiore non si trovava in Israele nell'antichità, anche se oggi due specie vi crescono spontaneamente. La sua identificazione esatta costituisce un problema difficile. Molto probabilmente si tratta del giglio bianco (Lilium candidum). Il grande commentatore biblico e grammatico medievale Abraham Ibn Ezra alludeva probabilmente a

7 Per un'informazione ulteriore sulla storia e la biologia della vegetazione arborea dell'Israele antico ricordata in questa ricorrenza, è utile anche <http://www.kkl-jnf. org/files/education/downloads/tubishvat/Trees_of_Israel_Italian.pdf > .

8 Si ritrova fra l'altro diverse volte in 1 Re, 7, 2 Cronache 4, nei salmi 45 e 69, ripetutamente nel Cantico dei Cantici, in Osea. 


\section{Ocula ${ }^{23}$}

Vol 21, No 23 (July 2020) • DOI: 10.12977/ocula2020-28

Ugo Volli | La rosa di Sharon. Fiori e piante nella tradizione ebraica antica

questo fiore quando affermò nel suo commento al secondo capitolo del Cantico che il nome shoshanah deriverebbe dalla parola ebraica shesh, che significa sei: "È un fiore bianco dal profumo dolce ma narcotico, e riceve il suo nome perché il fiore ha, in ogni caso, sei petali [shesh], all'interno dei quali sono sei lunghi filamenti”.

Un secondo nome sembra una specificazione del primo: shoshanat ha'emakim ("rosa delle valli"), menzionato ancora nel Cantico dei Cantici, ma questo non può riferirsi al giglio bianco poiché il giglio bianco non cresce nelle valli. Sembra essere il narciso, un fiore profumato che fiorisce nelle valli con un terreno pesante. Il terzo è lo havatzelet hasharon (rosa o giglio dello Sharon, che è la pianura costiera). È generalmente accettato che questo fiore sia identificato con il Pancratium Maritimum - una pianta bulbosa con fiori bianchi e molto profumati che fiorisce alla fine dell'estate nella pianura costiera. In realtà le due espressioni sono presenti accoppiate nell'autodefinizione della Sulamita e nella risposta del suo amato ai versi 2.1-2 del Cantico, secondo la procedura del raddoppiamento per variazione che è caratteristico della poesia ebraica. Guido Ceronetti, nella sua bella versione del 1975, traduce:

1. Io, l'asfodelo della pianura / il giglio degli acquitrini

2. Come tra i cardi la rosa / è tra le femmine l'amica mia

Enrica Salmaneschi (1982) scrive:

1. Io narciso della pianura, anemone delle valli

2. Come anemone fra le spine, così l'amica mia fra le donne

La versione compresa nella Bibbia cristiana delle Edizioni Paoline, firmata da Dalmazio Colombo e rivista da Gianfranco Ravasi e Primo Gironi dice invece:

1. Io sono un narciso di Saron, un giglio delle valli

2. Come un giglio fra i rovi, così è l'amica mia fra le giovani.

Aggiungo anche l'ultima traduzione italiana uscita di Piero Capelli: 9

Sono un asfodelo di Sharon, / un giglio delle valli!

Come una rosa tra i rovi / è la mia ragazza tra le ragazze.

Vale la pena di aggiungere anche la versione inglese della Jewish Publication Society, la più diffusa nel mondo ebraico:

1. I am a rose of Sharon, A lily of the valleys.

9 Benché sia un testo molto complesso ed enigmatico, o forse proprio per questo, le traduzioni abbondano. A quelle che ho citato va aggiunta certamente anche quella di Giovanni Garbini (Peideia 2010). 


\section{Ocula ${ }^{23}$}

Vol 21, No 23 (July 2020) • DOI: 10.12977/ocula2020-28

Ugo Volli | La rosa di Sharon. Fiori e piante nella tradizione ebraica antica

2. Like a lily among thorns, So is my darling among the maidens.

Bisogna notare che la seconda espressione (shoshanat ha'emakim) - si tratti di una rosa, di un giglio o di un anemone, dimorante fra rovi, spine o cardi -, è poi usata come attributo elogiativo anche al seno della sulamita (4:5) e al suo ventre (7:3); l'amante "bruca" questi fiori e li raccoglie (2:16, 6:2-3); ma anche le sue labbra sono paragonate al fiore $(5: 13) .{ }^{10}$ La confusione, anche solo quella botanica senza entrare nelle avventure dell'interpretazione morale e teologica che hanno sempre investito pesantemente il Cantico, è grande. Le note dell'edizione americana (Fishbane 2015) identificano tre principali identificazioni del primo fiore (havatzelet). Per il Targum (l'antica traduzione interpretativa della Bibbia in aramaico) per il primo filosofo ebraico Saadia Gaon, autore di una traduzione araba e poi anche per Jonah Ibn Janah, un grammatico ebreo di Cordova dell'XI secolo, si tratta del narciso; una seconda corrente interpretativa ne fa un Colchinum (<https://it.wikipedia.org/wiki/ Colchicum $>$ ), altri ancora la vedono come un croco.

Per quanto riguarda la shoshanah, Ibn Ezra e Emanuel Romano (autore del XIV secolo) ne farebbero una rosa, probabilmente per le spine, mentre altri parlano di "giglio nero". Il più autorevole commentatore ebraico della Bibbia, Rashi (vissuto in Francia nell'XI secolo), ha rafforzato l'identificazione della shoshanah con la rosa nel suo commento su questi versi dove afferma che resta sempre rossa. Altre interpretazioni moderne lo interpretano come un tulipano oppure "Hibiscus syriacus, Hypericum calycinum, Lilium candidum, Pancratium maritimum, Narcissus tazetta, Tulipa Montana and Tulipa sharonensis" (<http://www.flowersinisrael.com/RoseofSharon_page. htm>).

Senza procedere oltre in questo labirinto botanico, vale la pena di accogliere il suggerimento per spiegare questa confusione che viene da Paul Romanoff (1944) in un articolo dedicato ai temi iconografici delle monete ebraiche antiche:

Questa apparente inesattezza è spiegata dal termine generico shoshan che avrebbe potuto includere fiori come il loto e persino la rosa. In effetti, il Midrash contiene alcuni passaggi che parlano di un giglio morbido, e l'esempio di questo tipo è il mughetto, in parallelo alla rosa della valle. Oltre a queste allusioni, il Midrash menziona specificamente uno shoshanah - una rosa-giglio - che cresce nei frutteti, questa specie di rosa-giglio è il simbolo di Israele. Il giglio, shoshanah, è usato genericamente, poiché abbracciava altri fiori affini. I gigli crescevano sulle colline e nei campi. I gigli più scelti erano quelli che crescevano nelle valli, in prossimità dell'acqua. Perah - fiore nella Bibbia - viene spesso reso nel Targum con shoshanah -giglio. [...]

Si può certamente pensare che si tratti in partenza di terminologie generiche, che il testo intenda semplicemente parlare di fiori piacevoli, senza iden-

10 Rimando ai commenti in calce a Ceronetti (1975) (significativamente intitolato "Le rose del Cantico") e di Salmaneschi (1975) per un'analisi testuale. Gli stessi possono dare informazioni sull'interpretazione teologica molto poco letterale del Cantico. 


\section{Ocula ${ }^{23}$}

Vol 21, No 23 (July 2020) • DOI: 10.12977/ocula2020-28

Ugo Volli | La rosa di Sharon. Fiori e piante nella tradizione ebraica antica

tificarli ulteriormente. Ma questa possibilità non ci esime dall'interrogarci sul referente botanico primario, da cui il significato si sarebbe espanso poi a coprire tutto il campo semantico. Probabilmente non vi è modo di identificarlo, come per molti altri termini naturalistici che si ritrovano nella Bibbia ebraica, per esempio quello del mollusco che dava l'azzurro delle frange rituali o di certe specie dichiarate commestibili o interdette, e su cui già il Talmud spesso si interroga, ma questo non ci esime dal porci il problema.

$\mathrm{Al}$ di là dell'incerto riferimento botanico, quel che emerge dai versi del Cantico e da Osea è un nucleo semantico forte, che è lo stesso presente nel mondo occidentale moderno: i fiori rappresentano la bellezza, la grazia, il godimento che viene dal profumo, dal colore, dalla forma. Lo dice pochi versi più in là (2: 11-13) lo stesso Cantico:

11 Ecco, l'inverno è passato, è cessata la pioggia, se n'è andata;

12 i fiori sono apparsi nei campi, il tempo del canto è tornato e la voce della tortora ancora si fa sentire nella nostra campagna.

13 Il fico sta maturando i primi frutti e le viti in fiore spandono profumo. Alzati, amica mia, mia bella, e vieni, presto!

Il luogo in cui questo apprezzamento emerge nella maniera più chiaramente simbolica è il brano del libro dell'Esodo (25:31-38) in cui si descrive la menorah, il candelabro d'oro che doveva splendere nel Tabernacolo e poi fu eretto nel tempio di Gerusalemme ed è diventato il più diffuso e importante simbolo dell'ebraismo, ben prima della stella di Davide (recente, come attesta Scholem 1971),${ }^{11}$ e oggi è il simbolo dello Stato di Israele:

31 Farai anche un candelabro d'oro puro. Il candelabro sarà lavorato a martello, il suo fusto e i suoi bracci; i suoi calici, i suoi bulbi e le sue corolle saranno tutti di un pezzo. 32 Sei bracci usciranno dai suoi lati: tre bracci del candelabro da un lato e tre bracci del candelabro dall'altro lato. $33 \mathrm{Vi}$ saranno su di un braccio tre calici in forma di fiore di mandorlo, con bulbo e corolla e così anche sull'altro braccio tre calici in forma di fiore di mandorlo, con bulbo e corolla. Così sarà per i sei bracci che usciranno dal candelabro. 34 Il fusto del candelabro avrà quattro calici in forma di fiore di mandorlo, con i loro bulbi e le loro corolle: 35 un bulbo sotto i due bracci che si dipartano da esso e un bulbo sotto gli altri due bracci e un bulbo sotto i due altri bracci che si dipartano da esso; così per tutti i sei bracci che escono dal candelabro. 36 I bulbi e i relativi bracci saranno tutti di un pezzo: il tutto sarà formato da una sola massa d'oro puro lavorata a martello. 37 Farai le sue sette lampade: vi si collocheranno sopra in modo da illuminare lo spazio davanti ad esso. 38 I suoi smoccolatoi e i suoi portacenere saranno d'oro puro. 39 Lo si farà con un talento di oro puro, esso con tutti i suoi accessori.

11 Sulla Menorah e sul suo affermarsi come simbolo dell'ebraismo, rimando a Hachlili (2018). 


\section{Dcula ${ }^{23}$}

Vol 21, No 23 (July 2020) • DOI: 10.12977/ocula2020-28

Ugo Volli | La rosa di Sharon. Fiori e piante nella tradizione ebraica antica

I significati simbolici di questo oggetto sono stati molto discussi dai commentatori, soprattutto in riferimento alle luci, al loro numero e alla loro posizione, al senso da dare alla molteplicità dei bracci della lampada e all'unità richiesta nella loro fusione in un solo pezzo; quel che ci interessa è la decorazione floreale, che evidentemente intende portare un momento di bellezza al culto, ricordando la meravigliosa, precoce fioritura dei mandorli ai primi accenni della primavera, che nella regione di Israele spesso coincide con la data del Tu Bishvat di cui ho parlato. La decorazione floreale, in particolare di gigli, fu poi usata largamente per il Tempio, come si legge in 1 Re 6:32, 7:18, 22, 26, 39 ed è attestato ancora oggi da numerosi resti archeologici e numismatici che si sono conservati.

Paradossalmente è proprio questo carattere godibile dei fiori che ne interdice nel mondo ebraico uno degli usi più caratteristici fra i cristiani, quello di adornare feretri e cimiteri. Usarli per le tombe violerebbe infatti il principio per cui tutti i defunti sono uguali, portati alla sepoltura solo con un velario bianco, ma anche la proibizione di ba'al tashchit, dello spreco delle risorse. Poiché i morti non possono godere vista e profumo dei fiori, sarebbe sbagliato e perfino insultante fargliene dono. Com'è noto l'uso ebraico prevede che sulle tombe dei defunti cui si fa visita si ponga una pietruzza, come a consolidare il tumulo e il ricordo.

A questo apprezzamento si oppongono due difficoltà. Una è l'idea che i gigli nascano soprattutto in luoghi paludosi, da acque stagnanti e impure. Ne parla la Mishnah, lo strato più antico del Talmud, nel trattato Tohorot (purità) discutendo (3:7) il caso di "un bambino trovato accanto a una tomba con un mazzo di gigli in mano" e affermando che "i gigli nascono solo in luoghi sporchi", ma questo giudizio non è ripreso in seguito nella tradizione, salvo forse il caso della mitica bestia behemot, che in Giob. 40: 22 è ritratta mentre "si sdraia sotto le piante di loto, nel folto del canneto e della palude".

Più interessante è la seconda difficoltà, che è diffusa in molti passaggi biblici come Is 5:24, 18:4-5, 40:7, 80, Giob 14:2, 15:31-33. Prendiamo per esempio il Salmo 103:15-16:

15 I giorni dell'uomo sono come l'erba; egli fiorisce come il fiore dei campi;

16 se lo raggiunge un colpo di vento esso non esiste più e non si riconosce più il luogo dov'era.

Il tema è qui quello della caducità, il carattere effimero dei fiori cui viene accostata la vita umana.

Non vi è insomma una ricca metaforica floreale nella tradizione ebraica, come non vi ricorre un ricco uso materiale dei fiori nella liturgia e nelle pratiche di vita. Bisogna seguire però ancora almeno una traccia significativa nel territorio della mistica ebraica (la Kabalah, letteralmente "ricezione") Essa è dominata da un simbolo vegetale, l'“albero" delle sefirot (emanazioni o qualità o gradi del divino), un tema che è documentato a partire dal Sefer yezirah 


\section{Ocula ${ }^{23}$}

Vol 21, No 23 (July 2020) • DOI: 10.12977/ocula2020-28

Ugo Volli | La rosa di Sharon. Fiori e piante nella tradizione ebraica antica

(Libro della creazione, tradotto integralmente in Busi e Loewenthal 1998), scritto in Terra d'Israele fra il VI e il VII secolo. Questo tema è soggetto a molte diverse elaborazioni, proiezioni simboliche e schematizzazioni. ${ }^{12}$ La metafora dell'albero, secondo Busi (1998), è "divulgata dall'edizione cinquecentesca del Pardes rimmonim (il giardino dei melograni) di Moshè Cordovero", cabalista attivo a Safed in Israele nella prima metà del Cinquecento, e diventa ben presto diffusissima. Ma il rimando vegetale riguarda solo la forma approssimativa in cui le dieci sefirot sono sistemate in uno schema, che in qualche caso è letto anche come simile al corpo umano; e in esso non vi sono fiori.

\section{Havatzelet}

In questa tradizione però un'immagine floreale compare e con grande evidenza. È la havatzelet hasharon, la rosa della pianura (o qualunque altro genere vegetale essa sia), che ho discusso sopra. Proprio in apertura dello Zohar ([il libro dello] Splendore), che è di gran lunga il più importante testo della mistica ebraica, cui è riconosciuto uno statuto di libro quasi sacro, ${ }^{13}$ leggiamo:

Rabbi Chizkiya inizio a dire: "Come rosa tra le spine, così è la mia amica tra le fanciulle" (Cant. II, 2). Chi è la rosa? È la comunità di Israele. Come la rosa, che si trova tra le spine, ha in sé i colori rosso e bianco, così la comunità di Israele ha in sé il giudizio e la pietà. Come la rosa ha tredici petali, così la comunità di Israele ha tredici attributi di pietà, che la circondano da ogni parte. Anche Dio dal momento che fu ricordato per la prima volta fece scaturire tredici parole che ricordassero le comunità di Israele e la proteggessero: poi fu ricordato per la seconda volta. Perché il nome di Dio fu ricordato per la seconda volta? Per far scaturire le cinque foglie forti che circondano la rosa, che sono chiamate salvezze e costituiscono cinque porte. Di tale mistero è scritto: "Solleverò il calice delle salvezze" (Sal. CXVI, 13). Questo è il calice della benedizione, che deve essere poggiato sulle cinque dita, e non di più, come la rosa che si trova sulle cinque foglie forti, paragonate alle cinque dita. La rosa è dunque il calice della benedizione.

Com'è noto, il Cantico ha la forma di un poema d'amore molto terreno, in cui parlano in maniera non sempre chiaramente distinguibile due amanti molto appassionati che raccontano del loro amore. Ma la tradizione ebraica da un lato e quella cristiana dall'altro hanno interpretato questo testo in manie-

12 Nella ricchissima letteratura mi limito a rimandare a Scholem (1960) e Busi (1998).

13 Lo Zohar, secondo un uso consolidato dalla tradizione talmudica, si presenta come una discussione fra sapienti ebraici dell'epoca del dominio romano, organizzata come un'interpretazione del testo biblico. A partire da Scholem sembra stabilita la sua compilazione in ambienti ebraici della Castiglia nel tardo XIII secolo, e l'attribuzione della sua composizione definitiva al rabbino Moshè de Leon. Per una breve ma certamente informata discussione, cfr. Busi (1998: 70-75). Il testo è molto complicato, diviso in varie parti. Vi sono in italiano diverse traduzioni parziali la cui più completa è Leitman (2011). 


\section{Dcula ${ }^{23}$}

Vol 21, No 23 (July 2020) • DOI: 10.12977/ocula2020-28

Ugo Volli | La rosa di Sharon. Fiori e piante nella tradizione ebraica antica

ra fortemente simbolica: i maestri ebraici come una descrizione del rapporto (anche erotico e perfino matrimoniale, come mostra Idel 2005) fra Dio e Israele e quelli cristiani come una celebrazione della Chiesa. Limitandoci alla prima tradizione interpretativa, vale la pena di citare un detto di Rabbi Akiva, riportato nella Misnhà Yadaim (3:5):

Nessuno in Israele ha contestato che il Cantico dei Cantici [dicendo] che non contamina le mani [cioè che non è testo sacro]. Perché il mondo intero non è degno come il giorno in cui il Cantico dei Cantici fu dato a Israele; poiché tutti gli scritti sono santi ma il Cantico dei cantici è il santo dei santi.

Ogni suo dettaglio è significativo di questo rapporto e in particolare lo è la rosa (o anemone, o giglio). Essa dev'essere bianca e rossa, per riflettere "il giudizio e la pietà", che sono le due principali forme di manifestazione divina riconosciute dalla tradizione, corrispondente ai nomi "Elo-him" e al Tetragramma, che si usano tradurre nelle lingue occidentali, a partire dalla LXX, con termini equivalenti a "Dio" e "Signore"; deve avere tredici petali in corrispondenza ai "tredici attributi di pietà" che sono le clausole con cui la divinità si autodefinisce nel brano di Es 34:5-7, e che hanno grandissima importanza nella liturgia ebraica, perché vi si sottolinea la misericordia divina. ${ }^{14}$ Per questa complessa tematica dei nomi divini rimando al secondo e all'ottavo capitolo del mio Volli 2019, con la relativa bibliografia.

Si può certamente speculare sul fatto che l'uso teologico della "rosa di Sharon", con la pubblicazione dello Zohar avvenga pochi decenni prima del

14 Per capire la densità di questa metaforica e l'incrocio che essa opera di temi e luoghi diversi, per giungere poi a immagini molto nitide come quella della rosa dei tredici petali, vale la pena di riportare qui un altro passo dello Zohar che si riferisce al Cantico (I - 221a) : "Rabbi Shim'on iniziò a dire: "Io sono il giglio dello Sharon, la rosa delle valli profonde"» (Cant. II, 1). Come è cara la comunità di Israele al Santo, che benedetto egli sia, perché egli sempre la loda ed essa loda lui. Beato il possesso di Israele, che essi tengono in lui, nello speciale santo retaggio, come è scritto: "Poiché possesso del Signore è il suo popolo, Giacobbe è il suo speciale retaggio» (Deut. XXXII, 9). "Io sono il giglio dello Sharon". Questa è la comunità di Israele che sta come bellezza ed ornamento nel giardino dell'Eden. "Dello Sharon", perché canta e loda il re eccelso. Un'altra interpretazione dell'espressione "Io sono il giglio dello Sharon”. La comunità di Israele è il giglio che vuole abbeverarsi alle acque del torrente profondo, sorgente dei torrenti, come è scritto: «Ed il luogo arido diverrà un lago» (Is. XXXV, 7), "La rosa delle valli profonde", perché si trova nel luogo più profondo di tutti. Cosa sono i luoghi profondi? $\mathrm{E}$ ciò che è detto a proposito del verso: «Dalle profondità io ti invoco, o Signore» (Sal. CXXX, 1). "Il giglio dello Sharon" è il giglio di quel luogo irrigato, dal quale sgorgano i torrenti che non interrompono mai il loro flusso. "La rosa delle valli profonde" è la rosa di quel luogo che è chiamato il luogo più profondo di tutti ed è chiuso da ogni lato. Considera dunque. All'inizio essa è verde come il giglio, che ha le foglie verdi. Poi è "rosa", cioè rossa con tonalità bianche; è "rosa" con sei foglie; è "rosa" che si trasforma di colore in colore e muta i propri colori. "Rosa" - all'inizio è giglio, cioè quando desidera unirsi al re; quando poi si è unita al re, mediante il bacio, si chiama rosa, secondo ciò che è scritto: «le sue labbra sono rose» (Cant. V, 13). "Rosa delle valli profonde", perché muta i propri colori, talvolta per il bene, talvolta per il male; talvolta per la pietà, talvolta per il giudizio. 


\section{Ocula ${ }^{23}$}

Vol 21, No 23 (July 2020) • DOI: 10.12977/ocula2020-28

Ugo Volli | La rosa di Sharon. Fiori e piante nella tradizione ebraica antica

canto dantesco di Paradiso 31, 1-3: "In forma dunque di candida rosa / mi si mostrava la milizia santa / che nel suo sangue Cristo fece sposa”. La Commedia fu composta fra il 1304 e il 1321, lo Zohar, secondo le opinioni critiche contemporanee, fu redatto verso il 1275. Ma entrambi i testi riportano certo, anche sulle loro metafore della rosa, tradizioni precedenti. Per quanto riguarda l'ebraismo, il simbolo della rosa dai tredici petali e il rimando contestuale al Cantico è rimasto fra quelli più frequentati dalla tradizione, anche se non ulteriormente elaborato in maniera particolarmente attiva. Ultima sua notevole manifestazione è un libro molto diffuso di uno dei più popolari e creativi rabbini e teologi dell'ebraismo del Novecento, Adin Steinsaltz, che ha usato nel 1980 proprio questa immagine come titolo di un libretto sulla spiritualità e l'etica ebraica, che ha avuto grandissimo successo di letture, diffusione e traduzioni. I fiori dell'ebraismo sono pochi, ma persistenti e amati.

\section{Bibliografia}

Bahbout, Scialom

1986 Seder di Tu biShvat, Roma, Torah (ora visibile in <http://www.archiviotorah.it/libretti/seder_tubishvat.pdf $>$ ).

Berman, Joshua

2017 Inconsistency in the Torah: Ancient Literary Convention and the Limits of Source Criticism, New York, Oxford U.P.

2020 Ani Maamin: Biblical Criticism, Historical Truth, and the Thirteen Principles of Faith, Jerusalem, Maggid.

Bernheim, Pierre Antoine; Stavvrides, Guy

1991 Paradis, paradis, Paris, Plon.

Busi, Giulio

1998 La Qabbalah, Roma-Bari, Laterza.

Busi, Giulio; Lowenthal, Elena

1998 Mistica ebraica, Torino, Einaudi.

Carroll, James

2001 Constantine's Sword: The Church and the Jews, Boston, Houghton Mifflin.

Cassuto, Umberto

1941 Torath HaTeudoth, Jerushalaim, Magnes Press; trad. inglese The Documentary Hypothesis, Jerusalem, Shalem Press.

Celentano, Maria Silvana

1991 Il fiore reciso dall'aratro: Ambiguità di una similitudine (Catull. 11, 22-24), "Quaderni Urbinati di Cultura Classica" New Series, Vol. 37, No. 1.

Ceronetti, Guido

1978 Il cantico dei cantici, Milano, Adelphi.

Corbain, Alain 


\section{Ocula ${ }^{23}$}

Vol 21, No 23 (July 2020) • DOI: 10.12977/ocula2020-28

Ugo Volli | La rosa di Sharon. Fiori e piante nella tradizione ebraica antica

1982 Le miasme et la jonquille : L'odorat et l'imaginaire social aux XVIIIe et XIXe siècles, Paris, Flammarion.

Corradini, Matteo

2018 Il profumo dell'Eden, Firenze, Giuntina.

Fishabane, Michael

2015 The JPS Bible Commentary: Songs of Songs, Philadelphia, Un. Of Nebraska Press for JPS.

Gagliardi, Paola

2007 L'albero e il guerriero. Origine, funzione e storia di un topos omerico, "Silvae" 20 (<https://www.academia.edu/5630729/Lalbero_e_il_guerriero. Origine_funzione_e_storia_di_un_topos_omerico_Silvae_20_2007_pp._5$42>)$.

Goody, Jack

1993 The Culture of Flowers, Cambridge (UK), Cambridge University Press

1997 Representations and Contradictions: Ambivalence towards Images, Theatre, Fiction, Relics, and Sexuality, London, Blackwell.

Hachlili, Rachel

2018 The menorah, Leiden, Brill.

Idel, Moshè

2005 Kabbalah and Eros, New Haven, Yale University Press.

Kaplan, Stephen

1992 "Environmental preference in a knowledge-seeking, knowledge-using organism" in Barkow, J.; Tooby, J.; Cosmides, L. (editor) The Adapted Mind, Oxford, Oxford University Press, pp. 581-98.

Leitman, Micahel

2011 Zohar, Milano, Urra/Apogeo.

Maharil (Yaakov ben Moshe Levi Moelin )

1556 Minhagei Maharil, Sabbioneta.

Palewitz, Barry

2001 Why We Say It With Flowers, "The Scientist”, nov 2001.

Pinker, Steven

1997 How the Mind Works, New York, W.W. Norton \& Co.

Pollan, Michael

2001 The Botany of Desire: A Plant's Eye View of the World, New York, Random House.

Romanoff, Paul

1944 Jewish Symbols on Ancient Jewish Coins in The Jewish Quarterly Review, New Series, Vol. 34, No. 3 (Jan., 1944).

Salvaneschi, Enrica (a cura di)

1982 Cantico dei cantici, Genova, Il melangolo. 


\section{Dcula ${ }^{23}$}

Vol 21, No 23 (July 2020) • DOI: 10.12977/ocula2020-28

Ugo Volli | La rosa di Sharon. Fiori e piante nella tradizione ebraica antica

Scholem, Gersom

1960 Zur Kabbala und ihrer Symbolik, Zürich, Rhein Verlag.

1971 The Star of David: History of a Symbol, in The Messianic Idea in Judaism and Other Essays on Jewish Spirituality, New York, Schocken Books.

Steinsaltz, Adin

1980 The thirteen Petalled Rose, New York, Basic Books.

Volli, Ugo

2011 L'immaginario delle origini, "Lexia", 7-8.

2012 Domande alla Torà, Palermo, L’Epos.

2019 Il resto è interpretazione, Livorno, Belforte.

Wilson, Edward Osborn

1984 Biophilia, Cambridge, Mass., Harvard University Press.

Ugo Volli è Professore onorario presso il Dipartimento di filosofia e scienze dell'educazione dell'Università di Torino. Il suo ultimo libro è Il resto è interpretazione. Per una semiotica delle scritture ebraiche (Belforte 2019). 